Annales Geophysicae (2002) 20: 559-564 (C) European Geophysical Society 2002

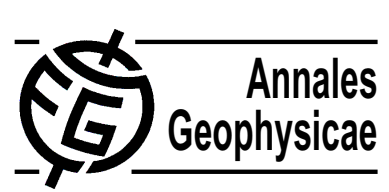

\title{
Surface ultraviolet radiation over east Siberia: seasonal variations
}

\author{
A. V. Mikhalev, M. A. Chernigovskaya, A. Yu. Shalin, and E. S. Kazimirovsky \\ Institute of Solar-Terrestrial Physics SD RAS, P.O. Box 4026, Irkutsk, 664033, Russia
}

Received: 10 May 2001 - Revised: 24 September 2001 - Accepted: 3 October 2001

\begin{abstract}
The results of spectral measurements of the daily near-noon surface direct solar ultraviolet radiation in the wavelength range of $295-345 \mathrm{~nm}$ obtained in Irkutsk (East Siberia) for the time interval of 1998-2000 are presented. For the period under consideration, the seasonal UV radiation variations are analysed that are associated with the total ozone dynamics, the transition of cyclonic and anticyclonic (Siberian anticyclone) periods, the presence of snow cover, and other factors. The analysis reveals an asymmetric behaviour of the seasonal course in ground-level UV radiation around the time of the summer solstice, with seasonal variation dependence on the wavelength. We have determined the irregular variations of surface UV radiation that is typical for the region, with their properties dependent on the season and on the spectral range analysed. The similarity of the above noted features from year to year was revealed.
\end{abstract}

Key words. Atmospheric composition and structure (Transmission and scattering of radiation; instruments and techniques) - Meteorology and atmospheric dynamics (middle atmosphere dynamics)

\section{Introduction}

An important problem in the study of solar-terrestrial relationships is the investigation of the possible variability of surface solar UV radiation. These variations can be associated with climatic changes of some of the atmospheric parameters observed during the last several decades, in particular, with the existence of negative temporal trends and/or long-term variations in the atmospheric ozone (Dobson, 1968; Udelhofen et al., 1999; Kruchenitsky et al., 1998; Kruchenitsky et al., 1999; Kazimirovsky et al., 1999). The variations in surface UV radiation (UVR) that are caused by changes in total ozone (TO) are in some cases comparable, or have even smaller values, compared with UV radiation variations of different time scales associated with other meteorological fac-

Correspondence to: A. V. Mikhalev (mikhalev@iszf.irk.ru) tors. The largest amplitude variations in the UV radiation are associated with changes in the angle of solar altitude (diurnal and seasonal variations), as well as the presence of a cloud cover. In mid-latitudes, these UVR variations can reach tens or hundreds of percent or more, depending on the wavelength. The seasonal variations can be divided into two categories. First, there are regular variations caused by seasonal changes in the angular altitude of the Sun (they depend on the latitude of the observation site). Second, the other category includes irregular variations associated with the properties of the climatic regime of a given region (i.e. they depend on its geographic location and on the physical-geographical properties). It is the irregular seasonal variations that are of special interest, as a climatic characteristic of the UV radiation field of the investigated region. These variations are also an important parameter that determines the variances of the monthly and annual amounts of UV radiation. The existence of the physical-geographic features of the investigated regions motivates the need for simultaneous observations of surface UVR in many regions of the planet (Bener, 1960; Bener, 1963; Justus and Murphey, 1994; McKenzie et al., 1993; Pu Bu Ci Ren et al., 1999; Svenoe et al., 1994; Udelhofen et al., 1999; Nezval, 1996; Mikhalev et al., 2000). The Antarctic region is of particular interest, where the most considerable TO variations are observed.

This paper presents the results of surface direct UVR observations at Irkutsk $\left(52^{\circ} \mathrm{N}, 102^{\circ} \mathrm{E}\right)$, the region of East Siberia. The characteristic property of the observation point is that the Siberian region is a zone of the stable Asian anticyclone in which anomalously low values of the TO have been observed lately in some periods (Kruchenitsky et al., 1998; Kruchenitsky et al., 1999; Mikhalev et al., 2000).

\section{Equipment and measurement procedure}

Daily measurements of the near-noon UVR values were made in the wavelength range of $296-346 \mathrm{~nm}$ using a spectrometer developed based on of the "KSVU-12" (LOMO) 
Table 1. The relative contribution of the diffused radiation into the measured radiation under different angular altitudes of the Sun

\begin{tabular}{c|c|c}
\hline $\begin{array}{c}\text { Angular altitude of } \\
\text { the Sun }\left({ }^{\circ}\right)\end{array}$ & \multicolumn{2}{|c}{$F_{g} \cdot \delta(\theta) \cdot d(\lambda, \theta)$} \\
\cline { 2 - 3 } $\mathbf{3 0 9} \mathbf{~ n m}$ & $\mathbf{3 4 4} \mathbf{~ n m}$ \\
\hline 15 & $\sim 1$ & 0.19 \\
30 & 0.19 & 0.06 \\
45 & 0.02 & 0.03 \\
60 & 0.01 & 0.02 \\
\hline
\end{tabular}

complex and the IBM AT computer. The spectrometer includes a monochromator with a reflecting rotating diffraction grating. The monochromator is designed using the asymmetric Fasti configuration with parabolic mirror objectives. The feature of the grating is $1200 \mathrm{rules} / \mathrm{mm}$. In order eliminate the scattered light inside the monochromator, is located in front of the entrance. The filter transmits only the UV spectral region under investigation. A photomultiplier is installed on the output of the monochromator. The stability of the measuring channel is monitored every day using a reference light source. All measured data are updated using this relative calibration. The measurements were made regardless of sky condition, i.e. on both clear and cloudy days. The measurements were made at the Sun's passage of the optical axis of the system at the same time of day $(12: 30 \mathrm{LT})$. The intensity of radiation reaching the fixed vertical mat quartz area oriented in a southward direction was recorded. The angular half-width of the view field of the optical path in the vertical plane was $\sim 32^{\circ}$. The spectral resolution in the spectral scanning was about $0.2 \mathrm{~nm}$. The scanning was made at steps of $0.02 \mathrm{~nm}$, with the scanning rate of $\sim 0.33 \mathrm{~nm} / \mathrm{s}$. The banded tungsten lamp, with a $200 \mathrm{~W}$ quartz window installed at $70 \mathrm{~cm}$ from the input window, was used for the absolute calibration of system. In addition, the Bouger straight-line method was applied for matching the measured radiation to tabular values of the extraterrestrial solar UVR radiation in the wavelength range under investigation.

Absolute spectral intensities of the continuous radiation spectrum of the lamp SIRSh-8.5-200-1 were determined through calculation. A pyrometer was used to determine the temperature of the lamp's working radiation surface. The calibration of the wavelengths in the measured spectra was carried out using a spectral mercury lamp from mercury emission lines and in the ultraviolet spectral region.

The Bouger method was used to plot the dependencies of the decimal logarithm of the values of measured radiation (in arbitrary units) on the optical mass of the atmosphere. Upon extrapolating the resulting curves to the optical mass equal to zero, the measured values of UV radiation were set equal to the tabular values of the atmospheric radiation at a given wavelength. The data on the extra-atmospheric solar spectrum reported in Makarova at al. (1991) was used. As a result, we estimate the accuracy of determination of the absolute values of the recorded UV radiation to be about $15 \%$. The error introduced by the measuring channel does not exceed $2 \%$.
Table 2. A comparison of the measured and model calculated ratios of radiation at two wavelengths

\begin{tabular}{c|c|c|c}
\hline \multirow{2}{*}{ Wavelengths } & \multicolumn{2}{|c|}{ Calculation } & Measure- \\
& ment \\
\hline \multirow{2}{*}{$309 \mathrm{~nm}$} & $\mathrm{~S}\left(60^{\circ}\right) / \mathrm{S}\left(15^{\circ}\right)$ & $\mathrm{D}\left(60^{\circ}\right) / \mathrm{D}\left(15^{\circ}\right)$ & 80 \\
\hline \multirow{2}{*}{$344 \mathrm{~nm}$} & 80 & $\begin{array}{c}14.4(b=0.025) \\
15.6(b=0.05)\end{array}$ & 80 \\
\hline
\end{tabular}

Both direct radiation and a part of the scattered radiation are recorded in the UVR measurements in an arbitrarily oriented area using the instrument with a finite entrance aperture (i.e. from a limited area of the sky). For this reason, the measurement scheme required an estimation of the contribution from the direct $S$ and scattered $D$ radiation to recorded signals, plus a selection of the processing technique for signals in order to subsequencly analyze them. A formula was obtained for determining the proportion of direct radiation in the recorded UVR:

$S_{\perp}(\lambda, \theta) / J_{r e c}(\lambda)=1 /\left\{K(\theta, \varphi)\left[1+F_{g} \cdot \delta(\theta) \cdot d(\lambda, \theta)\right]\right\}$.

Here, $S_{\perp}(\lambda, \theta)$ is a direct radiation incident on an area oriented normal towards the sunward direction, $J_{\text {rec }}(\lambda)$ is the radiation recorded by the instrument under the conditions described above, $K(\theta, \varphi)$ is the angular sensitivity coefficient of the receiving channel, $F_{g}$ is the geometrical coefficient determined as part of the celestial hemisphere within the instrument's field of view, and $\delta(\theta)$ is the coefficient characterizing the brightness dependence of the part of the sky with the distance from the solar disk on the sight axis by an angle $\theta$. The coefficient $d(\lambda, \theta)$ shows the proportion of scattered radiation relative to direct radiation in the UV range at different altitudes of the Sun, surface albedo and atmospheric turbidity.

The coefficients $K(\theta, \varphi)$ and $F_{g}$ were determined experimentally from a point source. The coefficient $d(\lambda, \theta)$ was calculated using the data reported in Belinsky et al. (1968). For estimations, the value of the coefficient $\lambda(\theta)$ was taken to be 1 for $\theta=15-30^{\circ}$ and 0.5 for $\theta=45-60^{\circ}$.

In the case where $F_{g} \cdot \delta(\theta) \cdot d(\lambda, \theta)$ becomes substantially smaller than 1 , this expression takes the form:

$S_{\perp}(\lambda, \theta)=J_{\text {rec }} / K(\theta, \varphi)$.

Table 1 presents the values of $F_{g} \cdot \delta(\theta) \cdot d(\lambda, \theta)$ estimated for different values of the angular altitude of the Sun at the two wavelengths of 309 and $344 \mathrm{~nm}$. It is evident from Table 1 that the contribution of scattered UVR at the $344 \mathrm{~nm}$ and $309 \mathrm{~nm}$ wavelengths in the range of solar altitudes at 30$60^{\circ}$ can make up $2-6 \%$ and $1-19 \%$, respectively. Consequently, under the given conditions, the contribution of scattered radiation can be neglected, and $J_{\perp}(\lambda, \theta)=S_{\perp}(\lambda, \theta)$ can be assumed. The contribution of scattered radiation to $J_{\perp}(\lambda, \theta)$ becomes substantial only in the case of minimum altitudes of the Sun at noon. The different rate of variation in the contribution from the scattered radiation depending on 


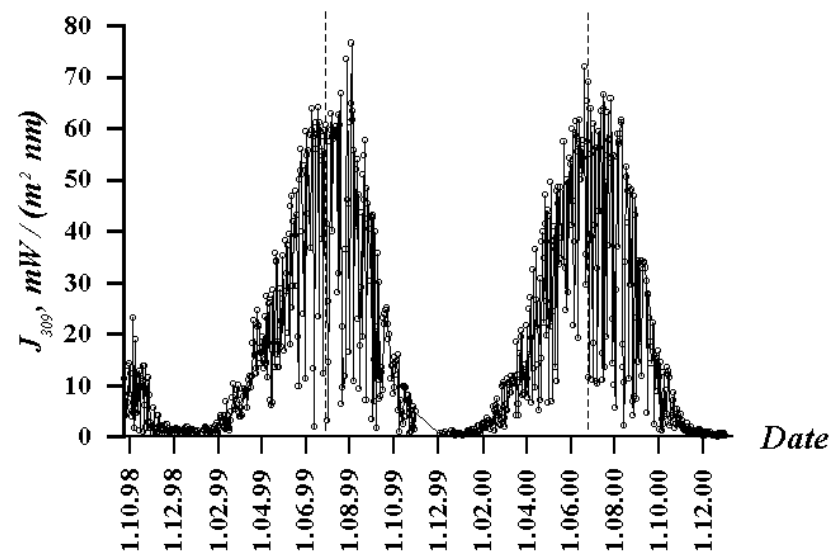

Fig. 1. The near-noon intensities of daily UVR observations on $309 \mathrm{~nm}$ wavelength.

the Sun's altitude at different wavelengths is caused by a different influence of the scattering and absorbing factors.

The values of $J_{\perp}(\lambda, \theta)$ obtained with the help of the procedure described above were compared with the calculated value variations of the direct and scattered radiation according to Belinsky et al. (1968) in the range of the measured angular altitudes of the Sun where $\theta \sim 15-60^{\circ}$. Table 2 presents the ratio values of the calculated (direct and scattered) radiation (Belinsky et al., 1968) to the measured radiation for two angles of solar altitudes at $15^{\circ}$ and $60^{\circ}$. The values of $\mathrm{D}\left(60^{\circ}\right) / \mathrm{D}\left(15^{\circ}\right)$ are given for two values of the coefficients (decimal) of atmospheric turbidity $b=0.025$ and $b=$ 0.05 (Belinsky et al., 1968). It is apparent from Table 2 that the observed variations of $J_{\perp}(\lambda, \theta)$ are consistent to a greater extent with the variations in direct radiation $S_{\perp}(\lambda, \theta)$. The values of $J_{\perp}(\lambda, \theta)$ are presented and analyzed later in this paper.

\section{Results of observations and discussion}

Figure 1 presents the results of daily UVR observations for a $309 \mathrm{~nm}$ wavelength spanning the time interval from 24 September 1998 to 31 December 2000. The vertical dashed line indicates the day of the summer solstice. It is evident from Fig. 1 that the annual UVR variation is clearly determined by the solar altitude. In addition, there is a strong day-to-day UVR variability, whose magnitude is affected primarily by the cloudiness variations, as well as by the aerosol conditions of the atmosphere and by the TO variations.

A more accurate analysis of the time variations reveals an asymmetric behaviour of the annual variation of UVR during the phases of spring growth (1st half-year) and autumn decline (2nd half-year), which are caused by variations in solar altitude, i.e. the presence of seasonal variations. For a numerical evaluation of this effect, Fig. 2 presents the averaged (over two years) UVR variations for two half-years (from 23 December to 22 June and from 23 June to 22 December). The curves are shown depending on the angular altitude of the Sun for the wavelengths of $309 \mathrm{~nm}$ (a) and $344 \mathrm{~nm}$ (b). The variations for the first and second half-years of 19992000 are shown by thin and thick lines, respectively. The values of $J_{\perp}(309 \mathrm{~nm}, \theta)$ for the second half-year in the range of the solar altitude at $30-60^{\circ}$ (June-September) consistently exceed those of $J_{\perp}(309 \mathrm{~nm}, \theta)$ for the first half-year (MarchJune) (see Fig. 2a) by as much as 40-50\%. The UVR intensity $J_{\perp}(344 \mathrm{~nm}, \theta)$ presented in Fig. $2 \mathrm{~b}$ shows an excess in the values for the first half-year of 1999-2000 over the respective values for the second half-year in the range of angles at $\sim 15-40^{\circ}$ (corresponding to the periods of JanuaryMarch and September-December). A substantial increase in $J_{\perp}(344 \mathrm{~nm}, \theta)$ in the range of angles of $30-40^{\circ}$ was observed in the spring period of 1999 .

The above differences of the seasonal dependencies of $J_{\perp}$ $(309 \mathrm{~nm}, \theta)$ and $J_{\perp}(344 \mathrm{~nm}, \theta)$ are caused by the peculiarities of the spectral dependence of the main factors influencing the incident UVR. It is known that in the spectral range under consideration in the case of a cloudless sky, the principal influence on the attenuation of UV radiation comes from the Rayleigh and aerosol scattering and from the absorption by the ozone. Furthermore, in the region of $\sim 345-350 \mathrm{~nm}$ wavelengths the main contribution to the radiation flux attenuation is made by the scattering from aerosols and by the Rayleigh scattering, while the proportion of ozone makes up $\sim 0.1 \%$. With a shift in the UV spectrum into the shortwavelength region, the contribution to the attenuation by the ozone increases up to $\sim 85 \%$ at the $280 \mathrm{~nm}$ wavelength (Banakh et al., 1986). According to our estimates, at the $309 \mathrm{~nm}$ wavelength, the contribution of ozone to the absorption at the ground will make up 50\%. In this case, the asymmetric behaviour of the seasonal UVR variation with respect to the summer solstice in the short-wavelength region of $309 \mathrm{~nm}$ can be caused by the pronounced annual variation of the TO over the region (Kazimirovsky et al., 1999) with a maximum in the first half-year. The behaviour of the UV radiation at the $344 \mathrm{~nm}$ wavelength seems to be associated to a greater extent with the difference in the cloud and aerosol regime of the atmosphere during the different seasons. By removing the annual trend of the UV radiation, it is possible to select irregular variations caused by climatic and meteorological properties of the region and by the prevailing regimes of atmospheric circulation at the point of observation for the different seasons (Shver et al., 1981).

Figure 3 shows the year-to-year changes in the irregular variations of the UV radiation at the $309 \mathrm{~nm}$ wavelength for 1999 and 2000. These curves were obtained by removing the annual trend of UV radiation and by subsequently smoothing the data using the moving average over 21 days. As expected, a recurrence of irregular variations was observed.

Figure 4 presents the averaged irregular UVR variations for two wavelengths of 309 and $344 \mathrm{~nm}$ for the year 1999. There is a visually traceable different degree of correlation of the UVR variations at the two wavelengths for the different seasons. The days between $\sim 100-280$ of the year (the angular altitude of the Sun where $\theta>30-40^{\circ}$ ) show a high similarity in the curves' form for the irregular variations of 

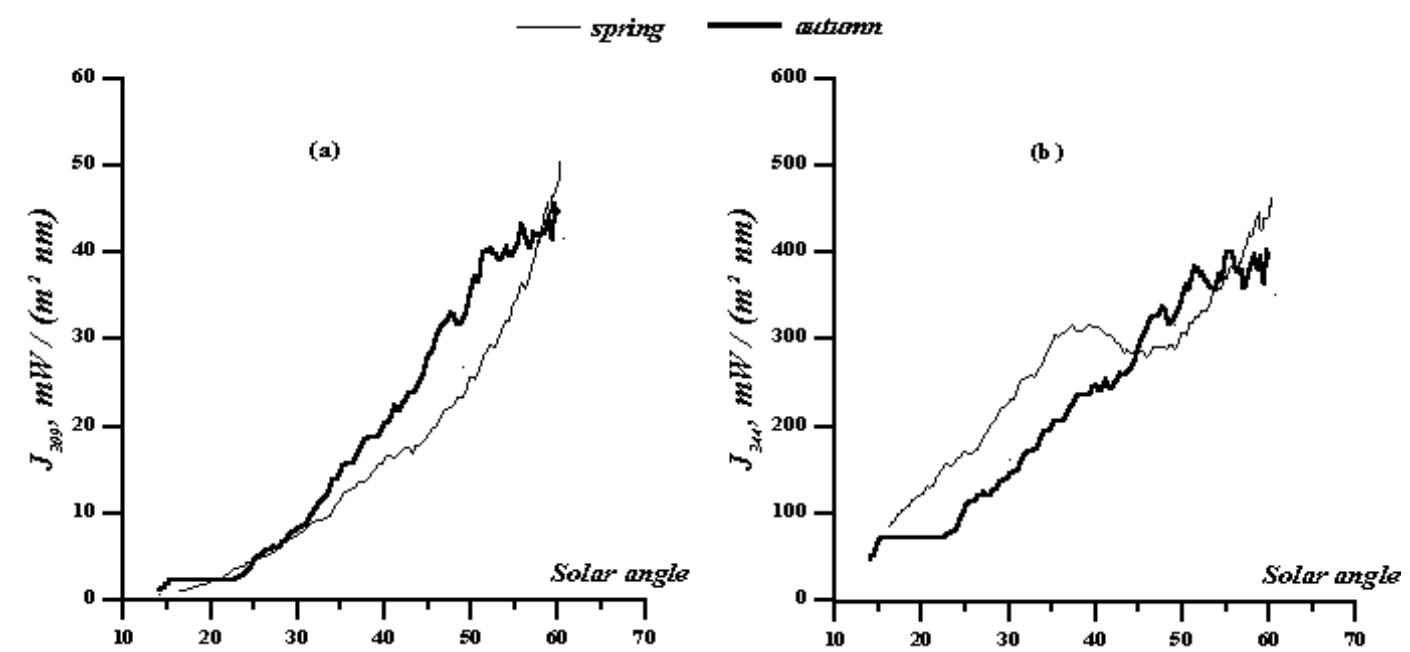

Fig. 2. The averaged, smoothed UVR variations for two half-years depending on the angular altitude of the Sun for the wavelengths of $309 \mathrm{~nm}(\mathbf{a})$ and $344 \mathrm{~nm}(\mathbf{b})$.

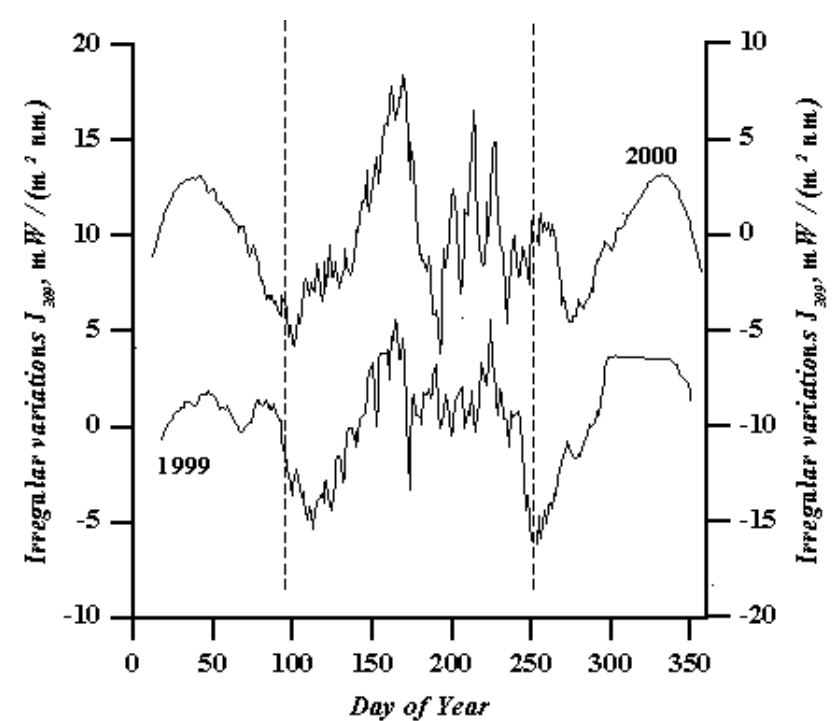

Fig. 3. The averaged year-to-year changes in the irregular UVR variations at $309 \mathrm{~nm}$ for 1999 and 2000.

the UV radiation for both wavelengths (with the presence of a high-frequency component). On the other hand, the days between $\sim 1-100$ and $\sim 280-365\left(\theta<44^{\circ}\right.$ and $\theta<30^{\circ}$, respectively) already show some differences in the UVR irregular variations as large as an anticorrelation (see, for example, the time interval of days between $\sim 1-80$ ). This peculiarity seems to be caused by a different contribution in the different seasons from the attenuation of the UVR radiation due to the Rayleigh and aerosol scattering and the absorption by ozone at different solar altitudes (optical masses). At low solar altitude, a larger contribution to the attenuation of direct radiation is made by the Rayleigh and ozone atmospheres (which are less variable compared to the aerosol component) due to a large optical mass value. At large angles of solar

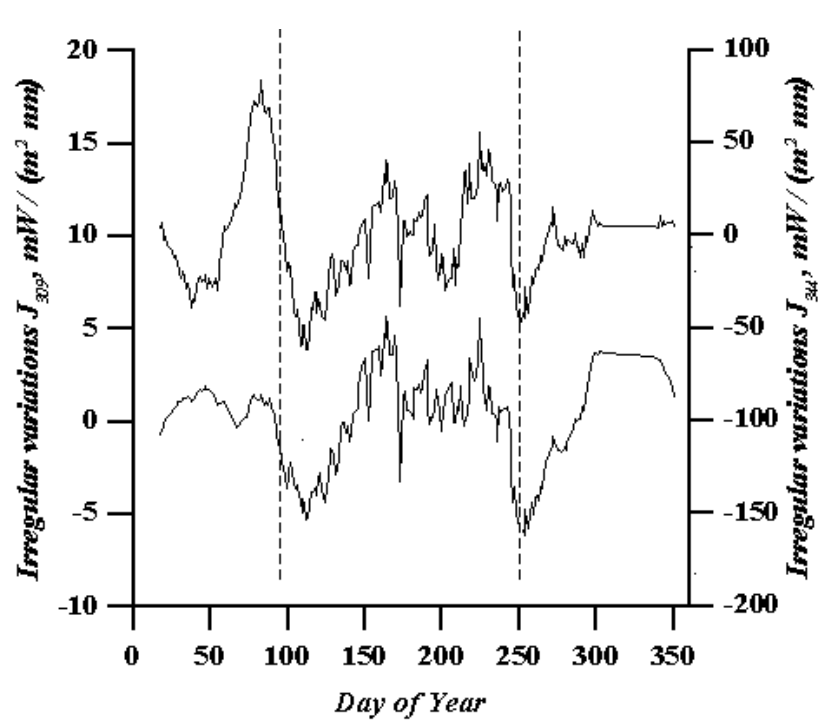

Fig. 4. The averaged irregular UVR variations upon removal of the annual trends for two wavelengths for 1999.

altitude and at small values of optical mass, variations in the aerosol component (and cloudiness) become crucial. Since this component has a weak spectral dependence of the scattering coefficients, this leads to a high correlation of UVR variations at the 309 and $344 \mathrm{~nm}$ wavelengths.

The presence of a distinctly pronounced annual TO variation in the region under consideration (Kazimirovsky et al., 1999), with a maximum at the beginning of the year and a minimum in autumn, is responsible for the difference in the irregular UVR variations. The UVR variations in the ozonedependent part of the spectrum $(309 \mathrm{~nm})$ differ from those in the long-wavelength part of the spectrum $(344 \mathrm{~nm})$, where the TO influence becomes insignificant. The same factor can be employed to account for the fact that the transition on the 


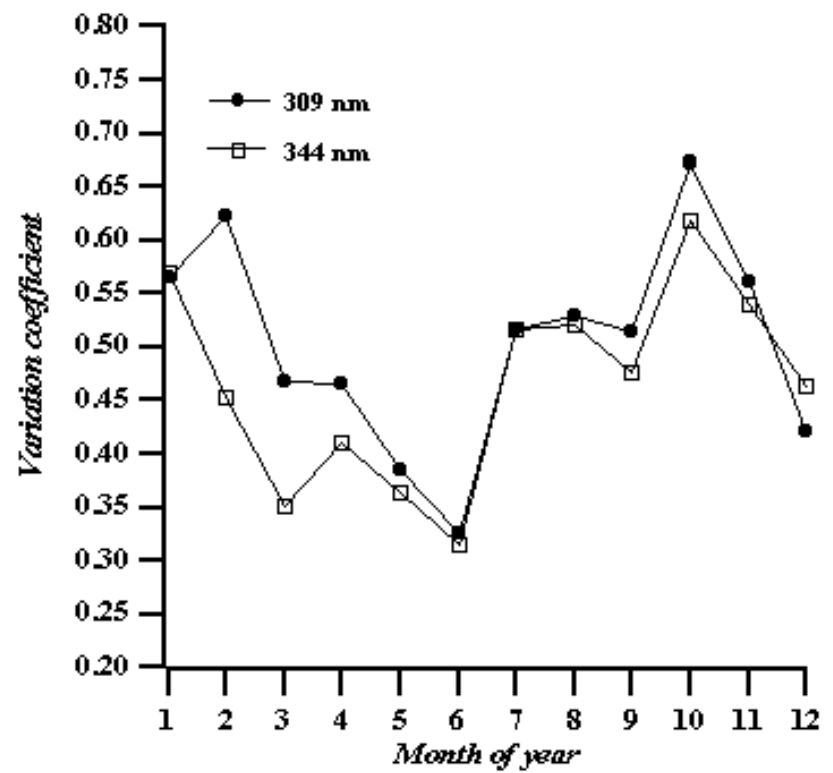

Fig. 5. The variation coefficients of the recorded UVR for the 309 and $344 \mathrm{~nm}$ wavelengths for the whole period of observation.

irregular variation curves from the sector with a high degree of correlation to the sector with a lower correlation occurs at different solar altitudes for UVR at two wavelengths. At the beginning of the year, when the TO has a maximum value this boundary can be determined near the solar altitude of $\sim 42-44^{\circ}$, and in autumn, near $30-32^{\circ}$ (in Figs. 3 and 4, these arbitrary boundaries are shown by vertical dashed lines).

The day-to-day variability of the near-noon surface UVR also has a seasonal dependence. Figure 5 presents the "variation coefficients" of the recorded UVR for the 309 and $344 \mathrm{~nm}$ wavelengths. These coefficients are determined as the averaged (over the same months during the period under consideration) ratio of the standard deviation to the monthly mean value of UVR. By excluding the winter months from the analysis (when the contribution of the scattered radiation to the value of $J_{\perp}(\lambda, \theta)$ becomes substantial), one notices that a maximum value of the variation coefficient is observed in October, and minimum value is observed in June. The variation coefficients for $309 \mathrm{~nm}$ wavelength in the first months of the year (February-April), when the largest values of TO are observed, exceed those for the $344 \mathrm{~nm}$ wavelength. This may be interpreted by the fact that the observed UVR variations in the ozone-dependent part of the spectrum are determined both by variations in the aerosol and Rayleigh components and by TO variations. On the other hand, in the summer months, the UVR variation coefficients at two analyzed wavelengths are virtually identical. This feature can be connected with the predominance of the contribution from variations in the aerosol atmosphere component, as described above.

Consequently, irregular variations of UVR in the different seasons of the year can be caused by different factors, such as TO dynamics, aerosol component, cloudiness, and have a different character for the different parts of the spectral range under consideration. The above mentioned peculiarities of the UVR variations must have differences in the other regions with different latitudes at the observation site, where a different TO dynamic, climatic and meteorological regime exists.

\section{Conclusions}

The main results of this study may by summarized as follows:

1. For the East Siberia region, we have identified an asymmetry of the seasonal variation of ultraviolet radiation $(295-345 \mathrm{~nm})$ with respect to the summer solstice. This asymmetry is brought about by the presence of a pronounced seasonal variation in the total ozone and by the meteorological properties of the region.

2. We have determined the irregular variations of surface UV radiation typical for the region and their properties dependent on the season and on the spectral range analyzed.

Acknowledgements. Topical Editor D. Murtagh thanks W. Josefsson for his help in evaluating this paper.

\section{References}

Banakh G. F., Ippolitov I. P., and Lopasova, T. A.: The atmospheric influence on the short-wavelength boundary of solar UV radiation at the Earth's surface, Kosmicheskie issledovaniya, (in Russian), 24, 6, 890-895, 1986.

Belinsky, V. A., Garadzha, M. P., Mezhennaya, L. M., and Nezval, E. I.: Solar and Sky Ultraviolet Radiation, Moscow: Izd-vo MGU, 228 (in Russian), 1968.

Bener, P.: Investigation on the spectral intensity of UV sky and sun + sky radiation, Davos, 1960.

Bener, P.: The duirnal and anneal variations of the spectral intensity of UV sky and global radiation on cloudless days at Davos, Davos, 1963.

Dobson, G. M. B.: Exploring the atmosphere, Oxford: Clarendon Press, 1968.

Justus, C. G. and Murphey, B. B.: Temporal trends in surface irradiance at ultraviolet wavelengths, J. Geophys. Res., 99, D1, 1389-1394, 1994.

Kazimirovsky, E. S., Matafonov, G. K., Vergasova, G. V., and Belinskaya, A. Yu.: Trends and low-frequency variations in total ozone content over East Siberia, Issledovaniya po geomagnetizmu, aeronomii i fizike Solntsa, (in Russian), 109, 2, 190-199, 1999.

Kruchenitsky, G. M., Kadyrova, T. V., Ivanova, N. S., and Zvyagintsev, M. A.: Total ozone content over Russia and adjacent territories in 1997, Meteorologiya i gidrologiya, (in Russian), 2 , 119-122, 1998.

Kruchenitsky, G. M., Zvyagintsev, M. A., and Ivanova, N. S.: Ozone content over Russia and adjacent territories in the 3rd quarter of 1999, Meteorologiya i gidrologiya, (in Russian), 11, 18-122, 1999. 
Makarova, E. I., Kharitonov, A. V., Kazachevskaya, T. V.: Solar Emission Flux, Moscow: Nauka, (in Russian), 397, 1991.

McKenzie, R. L., Kotkamp, M., and Seckmeyer, G., et al.: First southern hemisphere intercomparison of measured solar UV spectra, Geophys. Res. Lett., 20, 20, 2223-2226, 1993.

Mikhalev A. V., Chernigovskaya, M. A., Shalin, A. Yu., and Beletsky, A. B.: Spectral measurements of ultraviolet radiation in East Siberia during a total ozone content anomaly, Meteorologiya i gidrologiya, (in Russian), 3, 29-35, 2000.

Nezval, E. I.: Statistical characteristics of incident ultraviolet radiation at Moscow as deduced from data for 1968-1992, Meteorologiya i gidrologiya, (in Russian), 8, 64-71, 1996.

$\mathrm{Pu} \mathrm{Bu}$ Ci Ren, Gjessing, Y., and Sigernes, F.: Measurements of solar ultraviolet on the Tibetan Plateau and comparisons with discrete ordinate method simulations, J. Atmos. Terr. Phys., 61, 425-446, 1999.

Shver, Ts. A. and Formanchuk, N. P.: The Climate of Irkutsk, Leningrad: Gidrometeoizdat, (in Russian), 247, 1981.

Svenoe, T., Olsen, M., Henriksen, K., and Stamnes, K.: Measurements of lunar and solar ultraviolet spectra at high latitudes, Geomagn. and Aeron., 34, 5, 189-193, 1994.

Udelhofen P. M., Gies P., Roy C., and Randel, W. J.: Surface UV radiation over Australia, 1979-1992: Effects of ozone and cloud cover changes on variations of UV radiation, J. Geophys. Res., 104, D16, 19 135, 1999. 